\title{
Wearable Computing und RFID in Produktion und Logistik - Ansätze zur bereichsübergreifenden Nutzung digitaler Informationen
}

\author{
Prof. Dr.-ING. DiPL.-WI.-ING. W. A. GÜNTHNER \\ LEHRSTUHL FÜR FÖRDERTECHNIK MATERIALFLUSS LOGISTIK, TECHNISCHE UNIVERSITÄT MÜNCHEN \\ DIPL.-ING. M. WÖLFLE \\ LEHRSTUHL FÜR FÖRDERTECHNIK MATERIALFLUSS LOGISTIK, TECHNISCHE UNIVERSITÄT MÜNCHEN \\ DIPL.-ING. R. FISCHER \\ LEHRSTUHL FÜR FÖRDERTECHNIK MATERIALFLUSS LOGISTIK, TECHNISCHE UNIVERSITÄT MÜNCHEN
}

\begin{abstract}
Die voranschreitende Entwicklung von Konzepten und Systemen zur Nutzung digitaler Informationen im industriellen Umfeld eröffnet verschiedenste Möglichkeiten zur Optimierung der Informationsverarbeitung und damit der Prozesseffektivität und -effizienz. Werden die relevanten Daten zu Produkten oder Prozessen jedoch lediglich in digitaler Form zur Verfügung gestellt, fällt ein Eingriff des Menschen in die virtuelle Welt immer schwerer. Auf Grundlage dessen wird am Beispiel der RFIDTechnologie dargestellt, inwiefern digitale Informationen durch die Verwendung von in den Arbeitsablauf integrierten Systemen für den Menschen nutzbar werden. Durch die Entwicklung eines Systems zur papierlosen Produktion und Logistik werden exemplarisch Einsatzszenarien zur Unterstützung des Mitarbeiters in Montageprozessen sowie zur Vermeidung von Fehlern in der Kommissionierung aufgezeigt. Dazu findet neben einer am Kopf getragenen Datenbrille zur Visualisierung der Informationen ein mobiles RFID-Lesegerät Anwendung, mit Hilfe dessen die digitalen Transponderdaten ohne zusätzlichen Aufwand für den Anwender genutzt werden können.
\end{abstract}

The ongoing development of concepts and systems for the use of digital information in industrial environment opens different ways to optimize information processing and thus process effectiveness and efficiency. If relevant data on products or processes are only available in digital form a human intervention in the virtual world is getting more difficult. On this basis, the article shows with the example of RFID technology how digital information will be available for humans through the use of systems that are integrated in the workflow. By developing a system for paperless production and logistics we show exemplary use cases to support the employee in assembly processes and to avoid errors in order picking systems. In addition to a head-mounted display for the visualization of information, a mobile RFID reader is applied to make digital transponder data usable without additional expense to the user.

\section{Problemstellung}

\subsection{Der Mensch im Internet der Dinge}

Im Zuge der kontinuierlichen Verbreitung der RFID-Technologie - vor allem auch im logistischen Umfeld wird die Aufnahme und Verarbeitung der Daten innerhalb physischer (Logistik-) Prozesse für den Menschen zunehmend erschwert. Werden die Informationen zu Produkten oder Produktions- und Logistikprozessen ohne zusätzliche Visualisierung (z. B. mittels Etiketten) autark auf einem RFID-Transponder mitgeführt, findet der Mensch ohne zusätzliche Hilfsmittel (z. B. mobile/stationäre Datenterminals) keinen Zugang zu den digitalisierten Daten (vgl. Abbildung 1: Der Mensch im Internet der Dinge).

Durch die voranschreitende Digitalisierung der Informationsbereitstellung sowie die Vernetzung verschiedenster Objekte im „Internet der Dinge“ gilt es daher, zwangsläufig auftretende Medienbrüchen zwischen virtueller und realer Welt in erster Linie dort zu beherrschen, wo die Teilnahme des Menschen am System unumgänglich ist [Fle-05]. Gerade bei mobilen Anwendungen in Produktion und Logistik muss sichergestellt werden, dass dem Menschen trotz fortschreitender Technologie zu jeder Zeit und unter möglichst geringem Aufwand alle benötigten Informationen zur Verfügung stehen. Nur so kann der Mensch an den erforderlichen Stellen in die virtuelle Welt integriert werden. 


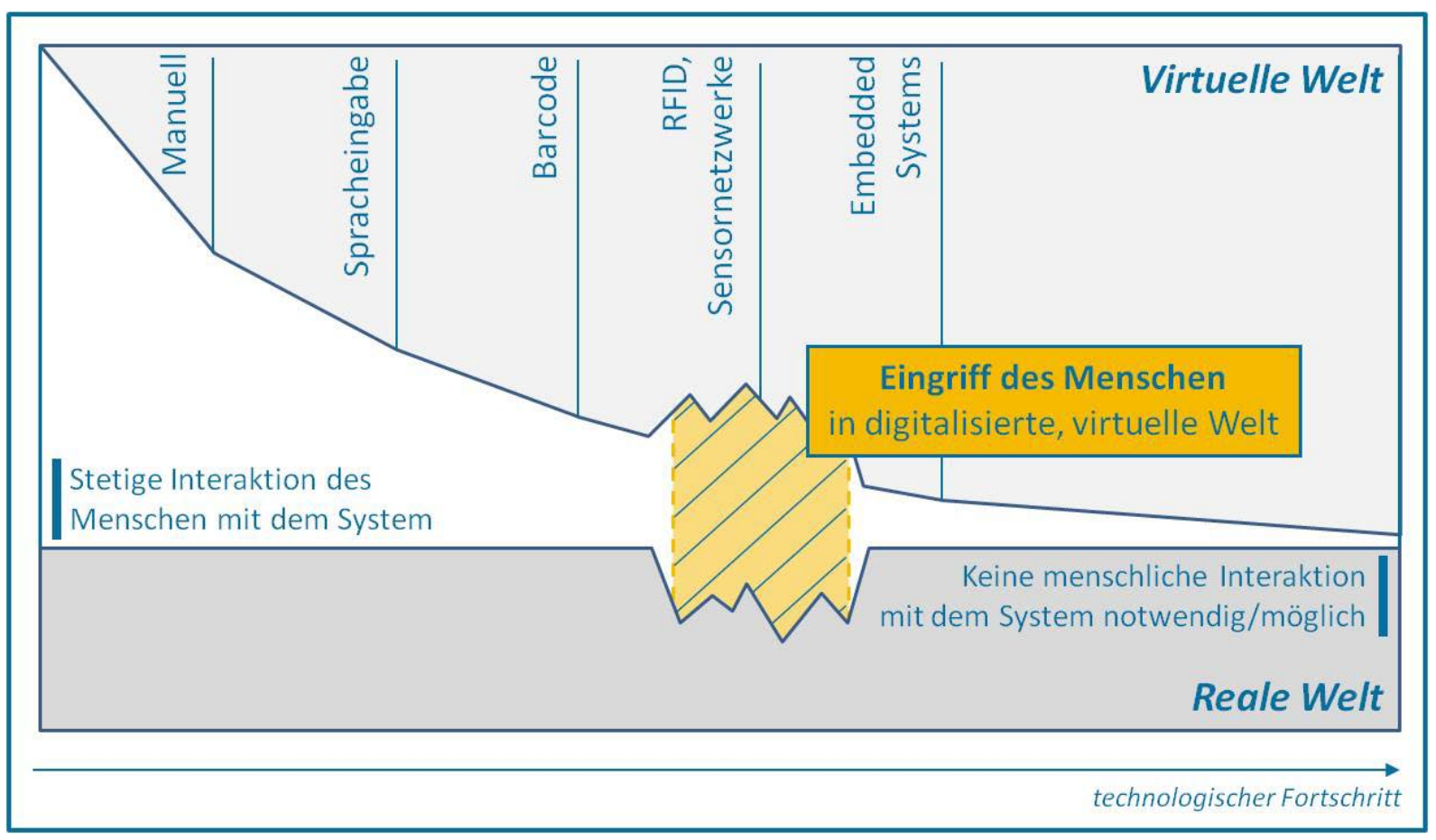

Abbildung 1: Der Mensch im Internet der Dinge (in Anlehnung an [Fle-05])

\subsection{Bereichsübergreifende Nutzung von Transpondern}

Obgleich RFID-Systeme immer mehr Anwendung in Produktion und Logistik finden, bleiben dennoch Hemmnisse und Unsicherheiten hinsichtlich einer möglichen Einführung der Technologie bestehen. So lässt sich die Nutzung des bereits applizierten Transponders über den primär verfolgten Unternehmensbereich und Einsatzzweck hinweg oftmals nicht oder nur mit erhöhtem Aufwand realisieren. Gerade eine bereichsübergreifende Verwendung der Transponder - egal ob auf Ladehilfsmitteln, Ladungsträgern oder Artikeln - kann jedoch einen erheblichen Teil der wirtschaftlichen Rentabilität des RFID-Projektes einnehmen. Aus diesem Grund gilt es, Wege und Methoden aufzuzeigen, inwiefern eine durch RFID-Transponder mitgeführte Information unter verschiedenen Rahmenbedingungen und innerhalb unterschiedlicher Prozessabläufe Verwendung finden kann. Die Beherrschung der Zusammenhänge, Abhängigkeiten und Wechselwirkungen, d. h. der Vernetzung des Informationsflusses zwischen einzelnen logistischen Prozessen steht dabei an oberster Stelle. Die Konzepte zur Identifikation der gekennzeichneten Objekte reichen von Pulkerfassungen an Wareneingangstoren bis hin zu mobilen Einzelerfassungen bei der Bewältigung logistischer Tätigkeiten. Eine übergreifende und durchgängige Nutzung der Transponderdaten unter Berücksichtigung menschlicher Eingriffe kann in letzter Konsequenz zur papierlosen Gestaltung sämtlicher Produktions- und Logistikprozesse führen.

\subsection{Lösungsansatz}

Neben einer fehlenden bereichsübergreifenden Nutzung der RFID-Transponder wird die Technologie häufig lediglich zur Erfassung und Interpretation der Daten genutzt. Durch die Verwendung der bereits vorhandenen Information für weitere Aufgaben (z. B. als Prüfmechanismus bei manuellen Tätigkeiten) lassen sich jedoch nicht nur zusätzliche Prozesszeiten reduzieren (z. B. durch den Wegfall administrativer Aufgaben), sondern ebenso die Qualität der Prozessabläufe erhöhen. Beide Aspekte können so zur wirtschaftlichen Absicherung der RFID-Anwendung führen. Zur technischen Umsetzung einer übergreifenden Nutzung digitaler Informationen aus RFID-Systemen existieren verschiedene Ansätze, welche insbesondere die Unterstützung des Menschen durch Arbeitsassistenzsysteme aufgreifen. Im Folgenden werden einige dieser Systeme aufgegriffen sowie ein System zur RFID-basierten Informationsbereitstellung mittels am Kopf getragener Displays vorgestellt. 


\section{Begriffsabgrenzung: Ubicomp, Ambient Intelligence (AmI), Wearable Computing}

Diskussionen um die Unterstützung und Entlastung des Menschen durch Assistenzsysteme im täglichen Leben (Gesundheit, Versorgung, Sicherheit etc.), aber auch in verschiedenen Bereichen des beruflichen Umfeldes sind allgegenwärtig. Verschiedene Ansätze verfolgen dabei das Ziel, die Nutzerfreundlichkeit und - gerade im Bereich der Arbeitsassistenzsysteme - auch die Nutzerakzeptanz durch eine strikte Reduzierung notwendiger Interaktionen zwischen Mensch und System zu erhöhen. Dadurch kann der Mensch möglichst unbemerkt und ohne zusätzlichen Aufwand bei seiner Tätigkeiten unterstützt werden. Der Anwender kann sich so stärker als zuvor auf seine Kernaufgabe konzentrieren, wodurch Arbeitsabläufe im industriellen Umfeld effizienter und weniger fehleranfällig gestaltet werden können. Zur Umsetzung dieses Gedankens existieren verschiedene Ansätze, welche sich im Wesentlichen im Grad der Integration in bestehende Arbeitsabläufe und der Mobilität unterscheiden.

\subsection{Ubiquitous Computing, Pervasive Computing}

In der Allgegenwärtigkeit des Computers (Ubiquitous Computing, Ubicomp) spiegelt sich der Ansatz wider, möglichst viele Objekte mit Intelligenz zu versehen und so Produkte und Produktionsmittel mit übergeordneten ERP-Systemen zu vernetzen. Neben verschiedenen Standards zur Datenübertragung finden dabei Datenträger auf Basis von RFID sowie unterschiedliche Sensoren Anwendung. Durch eine mobile Infrastruktur kommunizieren und interagieren die „smarten“ Objekte untereinander und passen sich weitgehend unbemerkt ihrer Umwelt an. Prozessrelevante Informationen lassen sich so in real-time erfassen, wodurch ganze Geschäftsprozesse und -modelle nachhaltig verbessert werden können ([Chr-03], [Lip-04]). Eine weiterführende, idealistische Sichtweise des Ubiquitous Computing mündet in der Definition des Pervasive Computing. Dabei rückt die Mobilität der Systeme in den Hintergrund, wobei der Ansatz verfolgt wird, den Computer in die Welt des Menschen zu integrieren. Der Rechner wird gezwungen, mit dem Menschen in der realen Welt zu leben, diesen zu unterstützen und dabei möglichst unentdeckt zu bleiben [Wei-91].

\subsection{Ambient Intelligence}

In Ergänzung zum Ansatz des Ubiquitous Computing beschäftigt sich Ambient Intelligence (AmI) verstärkt mit der Integration des Menschen in die virtuelle, digitalisiere Welt und verfolgt so das Ziel einer maximalen Akzeptanz des Nutzers. Durch eine optimierte Gestaltung der Schnittstellen zwischen Mensch und Computer stehen dabei die Anforderungen und Bedürfnisse des Menschen im Mittelpunkt. Die smarten Produkte werden auch bei diesem Ansatz möglichst nicht vom Nutzer wahrgenommen und unterstützen diesen bei seiner Tätigkeit durch eine bedarfsgerechte, kontextbezogene Informationsbereitstellung. Wie beim Ubiquitous Computing lässt sich Ambient Intelligence somit als eine humanzentrierte Technikvision beschreiben, welche sich verschiedenster Basistechnologien wie der Virtual und Augmented Reality, Hochfrequenztechnik, Sensorik etc. bedient und den Nutzer in das Zentrum der Kommunikation zwischen Mensch und Technik stellt ([Aar-04], [Did-10]).

\subsection{Wearable Computing}

Zur Realisierung der Ansätze aus Ubiquitous Computing, Ambient Intelligence o. ä. finden Wearable Computing Systeme Einsatz, welche sich im Vergleich zum reinen mobilen, tragbaren Computer als integrierte Systeme („Wearables“) auszeichnen. Dadurch wird der Anwender bei seinen Tätigkeiten unterstützt ohne bei der Durchführung seiner Kernaufgabe mit zusätzlichem Aufwand abgelenkt oder in seinen Aufmerksamkeit unnötig gebunden zu werden. Wearable Computing Systemen sollen auf diese Weise eigenständig spezielle Tätigkeiten oder Sequenzen innerhalb definierter (Arbeits-) Abläufe erkennen, um dadurch mit einer bedarfsgerechten Bereitstellung verschiedener, auf den Nutzer abgestimmter Dienste zu reagieren. Nach [Woo-03] können die Systeme auf Basis der PPaFF-Skala (Processor Performance and Function Flexibility) unterschieden werden. Dabei werden neben der Leistung des Prozessors und der Flexibilität der bereitgestellten Funktion unter anderem Faktoren wie der Energieverbrauch oder der Grad an erforderlicher Aufmerksamkeit durch den Anwender berücksichtigt. Beim Einsatz RFID-basierter Arbeitsassistenzsysteme, welche dem Nutzer durch mobile Lesegeräte den Zugang zu digitalisierten Informationen gewährleistet, spielen insbesondere die beiden zuletzt genannten Kriterien eine wesentliche Rolle. ([ScR-10], [Woo-03]) 


\section{Stand der Technik}

\subsection{Anwendungen RFID-basierter Ubicomp Solutions}

Bereits im Jahr 2001 berichtet [Fle-01] in seinen Ausführungen von den Roll-out-Planungen für ein RFIDbasiertes Ubicomp-System bei der Herstellung von Wafern. Die Intelligenz der RFID-Transponder auf den Platten soll neben der Positionsbestimmung der Wafer innerhalb des Fertigungsgebäudes genutzt werden, um die Wafer zu autarken Produkten zu entwickeln, die eigenständig Berichte über ihren aktuellen Status abgeben. Die Wafer versenden Warnungen via SMS wenn sie bspw. über einen längeren Zeitraum nicht bewegt wurden oder ihr avisierter Auslieferungstermin gefährdet ist.

Mit extrem hohen gesetzlichen Anforderungen an Qualität, Sicherheit und Dokumentation von Wartungsaufgaben bietet die Luftfahrtindustrie ein weiteres Anwendungsgebiet für Ubicomp-Systeme. Über einen mit RFID-Lesegeräten sowie geeigneten Systemen zur Datenübertragung ausgestatteten Werkzeugwagen werden automatisch Informationen zu den ,intelligenten“ Werkzeugen (bspw. über eine anstehende Wartung) an ein zentrales System gesendet. Mithilfe zusätzlicher, mobiler RFID-Lesegeräte können Mitarbeiter zudem sämtliche Daten zu den Werkzeugen abrufen, welche zur korrekten Bedienung bzw. zur Durchführung verschiedener Arbeitsabläufe relevant sind. ([Fle-01], [Lam-05])

\subsection{Wearable Computing Ansätze für mobile RFID-Lesegeräte}

Können RFID-Transponder prozessbedingt nicht über stationäre Identifikationspunkte erfasst werden, finden häufig mobile RFID-Lesegeräte auf Basis handelsüblicher Handhelds Verwendung. Parallel zur eigentlichen Haupttätigkeit bedeutet die Handhabung der Geräte bei Anwendungen in Produktion und Logistik jedoch meist einen erhöhten Aufwand. Das verfolgte Ziel liegt daher darin, ein mobiles RFID-Lesegerät nach den Ansätzen des Wearable Computing in die Tätigkeiten des Anwenders zu integrieren, ohne ihn dabei bei seiner Kernaufgabe zu stören. Seit einigen Jahren gibt es in der Forschung die Bestrebung, das RFID-Lesegerät in Form eines Handschuhs bzw. Armbandes zu etablieren (vgl. Abbildung 2: Gestaltung eines tragbaren RFIDLesegerätes). Aufgrund der meist geringen erforderlichen Lesereichweite sowie der Unempfindlichkeit gegenüber dem hohen Wassergehalt des menschlichen Körpers konzentrierten sich die Forschungstätigkeiten dabei zumeist auf niederfrequente RFID-Systeme. Die „University of Washington“ entwickelte in Kooperation mit Intel Research Seattle einen RFID-Handschuh im Frequenzbereich von 13,56 MHz zur Untersuchung von Aktivitäten des täglichen Lebens durch die Identifikation gekennzeichneter Objekte ([Phi-04], [Fis-05]).

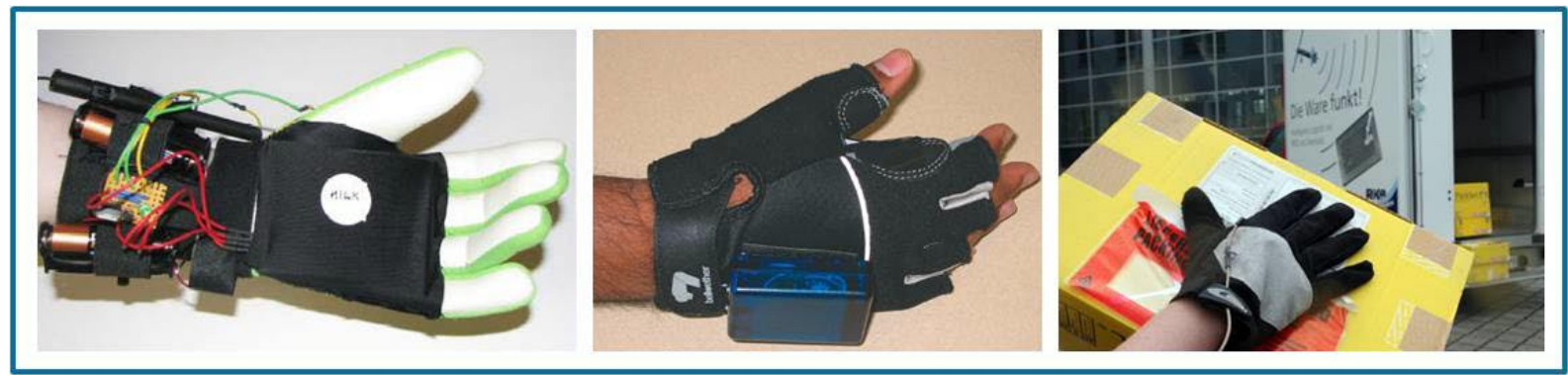

Abbildung 2: Gestaltung eines tragbaren RFID-Lesegerätes ([Lus-07], [Phi-04], [IFF-10])

Die Überwachung des körperlichen Zustandes sowie die Unterstützung der Selbstständigkeit von Menschen mit gesundheitlichen Problemen bspw. bei vermindertem Erinnerungsvermögen oder Blindheit stellen weitere Anwendungen für den Einsatz eines mobilen RFID-Handschuhs im Bereich des Gesundheitswesens dar ([Lus07], [Lee-10]). Weitere Forschungsaktivitäten beschäftigten sich mit dem Einsatz von RFID-Handschuhen in Wartungs- und Instandhaltungsprozessen ([ScA-00], [Wit-06]). Auch hier bringen niederfrequente RFIDSysteme die besten Voraussetzungen mit, da beim Lesevorgang meist mit direktem Kontakt zwischen Transponder und Antenne des Handschuhs gearbeitet werden kann. Jedoch auch auf dem in Produktion und Logistik weiter verbreiteten UHF-Frequenzband (EU: $868 \mathrm{MHz}$ ), welcher sich u. a. durch höhere Lesereichweiten auszeichnet, existieren bereits Entwicklungen von RFID-Handschuhen. Diese gehen jedoch wie auch im niederfrequenten Bereich nicht über den prototypischen Aufbau hinaus. Das Fraunhofer-Institut für Fabrikbetrieb und -automatisierung (IFF) beispielsweise arbeitet insbesondere im logistischen Umfeld an der Integration eines RFID-Handschuhs zur dezentralen Erfassung gekennzeichneter Ware [Kir-10]. 


\subsection{Industrielle Anwendungen für mobile Informationsbereitstellung}

Beim Einsatz mobiler RFID-Lesegeräte unter „Wearable Computing“-Aspekten bleibt die Darstellung der aus den Transpondern bzw. dahinterliegenden Datenbankstrukturen gewonnenen Informationen offen. Als direkte Schnittstelle zum Menschen ist das Visualisierungsmedium jedoch ein entscheidender Faktor, um den Menschen bedarfsgerecht und ergonomisch mit relevanten Informationen zu versorgen und so eine möglichst hohe Nutzerakzeptanz zu erreichen. Die Bereitstellung prozessrelevanter Informationen dezentral am Ort der Objektidentifikation beruht für Anwendungen in Produktion und Logistik derzeit häufig auf papierbasierten Systemen (Kommissionierliste, Montageanleitungen etc.). Für die Identifikation von Barcodes existieren zudem mobile Handlesegeräte in Form von Laserscannern mit integriertem Display oder mobile Computer mit angeschlossenem Fingerscanner. Einige dieser Handhelds können für den Einsatz von RFID auch um ein HFModul erweitert werden, wodurch eine mobile Datenbereitstellung auf Basis von RFID bereits realisiert werden kann. Anwendung im industriellen Umfeld finden solche Systeme in den meisten Fällen für Wartungs- und Inventurprozesse, welche ein mobiles Erfassen von Objekten voraussetzt. Als Beispiel dient hierbei die Wartung von Brandschutzklappen am Frankfurter Flughafen [VDI-10]. Dabei wird der vom Gesetzgeber vorgeschriebenen langjährigen Nachweispflicht Folge geleistet, welche sich als sehr schwierig realisierbar erwies und durch die manuelle Eingabe und Pflege der Daten ein hohes Fehlerpotenzial barg. Durch den Einsatz von RFID konnten nicht nur administrative Tätigkeiten reduziert, sondern zudem der Nachweis von Wartungsarbeiten optimiert werden. Mit harten Anforderungen an die Robustheit des System aufgrund von rauen Umgebungen setzen auch die Berliner Wasserbetriebe bei der Inventur ihrer Anlagen auf eine mobile Informationsbereitstellung auf Basis RFID-fähiger Handlesegeräte [Sma-10]. Ein RFID-System im 13,56 MHz Frequenzband ersetzt dabei manuelle Prüfvorgänge anhand von Papierlisten sowie die manuelle Eingabe der aktualisierten Daten ins unternehmensinterne ERP-System.

\subsection{Visualisierung der Informationen über head-mounted Displays}

Als Alternative zu papierbasierten Systemen sowie stationären oder handbasierten, mobilen Monitoren könnten zukünftig kopfbasierte Displays (auch: Datenbrillen oder head-mounted Displays, HMD) eine kontextbezogene Visualisierung prozessrelevanter Informationen unterstützen. Obgleich ergonomische Unzulänglichkeiten die Nutzerfreundlichkeit dieser Systeme gerade für Anwendungen in Produktion und Logistik einschränken und dadurch einen dauerhaften Einsatz häufig (noch) verhindern, bergen HMD verschiedene Potenziale bei der bedarfsgerechten und nahezu unbemerkten Unterstützung des Menschen. Gerade bei einer hohen Komplexität der Arbeitsaufgabe, einem erhöhten Anteil an Suchtätigkeiten oder ganz allgemein bei Arbeitsabläufen, die den Bedarf an einer informatorischen Unterstützung mitbringen, können durch die Parallelisierung von Informationsaufnahme und Nebentätigkeiten sowie die Verringerung des Suchaufwandes Einspareffekte erzielt werden ([Alt-03], [Tüm-08]). So entfällt bspw. das Mitführen und Handhaben von Papierbelegen, Handhelds etc., wodurch der Nutzer beide Hände zur freien Verfügung hat und sich somit verstärkt auf seine Haupttätigkeit konzentrieren kann. Verschiedene Kriterien wie Okularität, Bauart, Field of View, Auflösung oder Bildwiederholfrequenz lassen eine Unterscheidung der am Markt verfügbaren Datenbrillen zu [Rei-09]. Monokulare Displays haben gerade für industrielle Anwendungen den Vorteil, dass das Sichtfeld des Nutzer meist nur bedingt eingeschränkt wird, da mit dem zweiten Auge die Umgebung in voller Sehschärfe wahrgenommen werden kann. Weiter können die Datenbrillen nach Look-Through- (Durchsichtsysteme) und Look-Around-HMD (Umsichtsysteme) unterteilt werden. Look-Around-HMD haben den Vorteil, dass nur ein Teil der realen Umgebung durch das Display verdeckt wird, während der Nutzer von Look-Through-HMD seine Umwelt nur durch das HMD wahrnimmt und das gesamte Blickfeld vom HMD verdeckt wird [Rei-09].

Aktuelle Entwicklungen im Bereich der Datenbrillen, die als Bestandteil von Arbeitsassistenzsystemen Anwendung finden können, beschäftigen sich mit der Stärkung der Akzeptanz beim Endanwender durch eine verbesserte Systemergonomie und Usability mittels einer nutzerzentrierten Systemgestaltung [Tüm-08]. Zudem beschäftigt sich die Forschung aktuell mit Untersuchungen zur potentiellen Leistungssteigerung bzw. Fehlerreduzierung in Kommissioniersystemen, wobei HMD-basierte Systeme mit bereits ausgereiften Technologien wie Pick-by-Voice, Pick-by-Light, Papierliste etc. verglichen werden. Dabei konnten die Potenziale der Datenbrille zur Leistungssteigerung sowie zur Vermeidung von Fehler nachgewiesen werden ([Gün-09], [Ibe-09]). Weitere Einsatzfelder im industriellen Umfeld wie Montage, Wartung oder Qualitätssicherung versprechen ähnliche Potenziale.

\section{RFID-basierte Informationsbereitstellung mittels kopfbasierter Displays}

Werden die Ansätze des Wearable Computing für RFID-Lesegeräte nun mit dem Einsatz kopfbasierter Displays zur Visualisierung der Informationen kombiniert, lassen sich verschiedene Einsatzfelder innerhalb von (C) 2011 Logistics Journal: Not reviewed publications - ISSN 1860-5923

Seite 5 
Produktions- und Logistikprozessen identifizieren, welche durch eine nahezu unbemerkte Unterstützung des Werkers optimiert werden können. Ein solches Arbeitsassistenzsystem eröffnet durch eine ergonomische Integration der erforderlichen Komponenten in die Ausrüstung des Werkers den Weg zu einer papierlosen Datenbereitstellung. Zudem wird eine flexible, ortsunabhängige und kontextbezogene Bereitstellung und Visualisierung prozessrelevanter Informationen ermöglicht.

Kern des Systems ist neben dem HMD das mobile RFID-Lesegerät, welches in der Form eines Handschuhs am Handgelenk getragen wird. Dadurch wird es möglich, RFID-Transponder an Produkten, Ladungsträgern oder Lagerplätzen zu erfassen ohne dabei die gewohnte Bewegung des Werkers zu verändern. Somit stehen die benötigten Informationen ohne zusätzlichen Aufwand stets zur richtigen Zeit und am richtigen Ort zur Verfügung. Zur Navigation durch Bedienoberflächen für den Abruf von Hilfestellungen sowie die Eingabe aufgetretener Fehler beinhaltet das System ein zusätzliches Interaktionsgerät. Die statische Visualisierung prozessrelevanter Informationen kann zudem durch die Integration eines Trackingsystems zur Darstellung dynamischer, von der Orientierung des Nutzers abhängiger Zusatzinformationen erweitert werden (vgl. Abbildung 3: RFID-basierte Informationsbereitstellung mittels kopfbasierter Displays - Systemkomponenten).

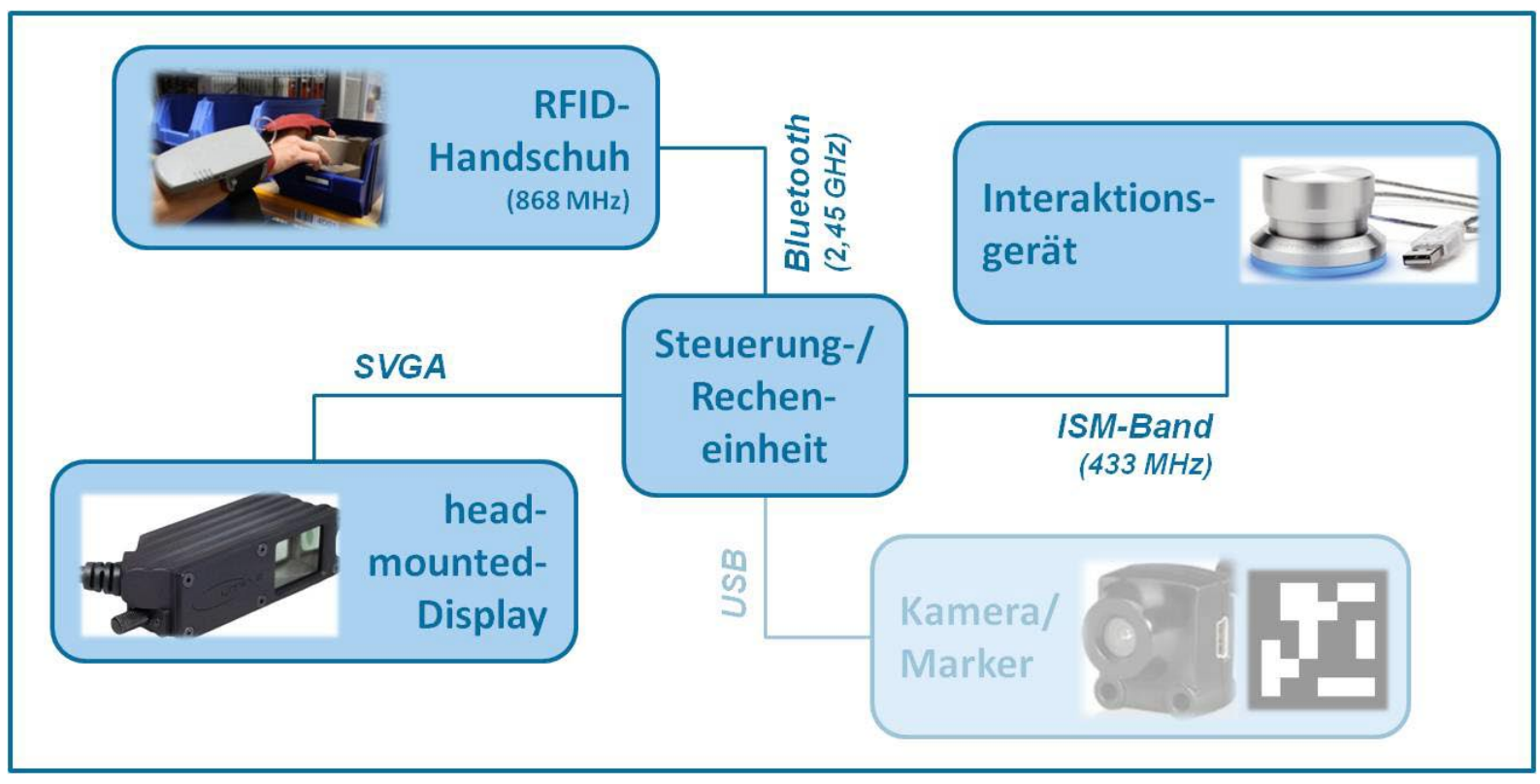

Abbildung 3: RFID-basierte Informationsbereitstellung mittels kopfbasierter Displays - Systemkomponenten

\subsection{RFID-Handschuh als mobiles Lesegerät}

In seiner Grundfunktion überträgt das mobile RFID-Lesegerät die aus den Transpondern gelesenen Daten über eine kabellose Schnittstelle (WLAN, Bluetooth etc.) an eine zentrale Recheneinheit. Dort werden nicht nur die relevanten Prozessinformationen gehalten, sondern auch die Auswertung und Interpretation der Transponderdaten durchgeführt. Zu den Komponenten des mobilen Lesegerätes zählen neben der Antenne und dem eigentlichen HF-Modul (inkl. Controller) dementsprechend lediglich eine Kommunikationseinheit sowie die Stromversorgung. Um die für eine erfolgreiche Identifikation der Transponder erforderliche Leistung und somit auch den Bedarf an Energie zu minimieren, gilt es die Richtcharakteristik der Reader-Antenne und die Anbringung der Transponder optimal aufeinander abzustimmen. Die Anforderungen ergeben sich dabei aus verschiedenen Bereichen: Den Eigenschaften der Reader-Antenne, dem gesamten Erfassungsszenario bestehend aus der Transponderanbringung und Umgebungsbedingungen - sowie der bereichsübergreifenden Verwendung der Transponder. Werden die Transponder beispielsweise an Behältern angebracht, um die mitgeführten Daten in verschiedenen Unternehmensbereichen zugleich zu verwenden, gilt es eine Position zur Anbringung der Transponder zu finden, welche eine erfolgreiche Identifikation in allen Bereichen ermöglicht. Zudem müssen verschiedene technische Rahmenbedingungen (RFID-Gate im Wareneingang, mobiles RFIDLesegerät in der Kommissionierung, Stationäre Lesegeräte in der Produktion etc.) berücksichtigt werden.

Im Sinne des Ambient Intelligence dient das mobile RFID-Lesegerät jedoch nicht nur zur reinen Identifikation der Transponder an Produkten, Ladungsträgern oder Lagerplätzen, sondern findet ebenso Verwendung als Interaktionsgerät zwischen Mensch und Maschine. Durch das nahezu unbemerkte und automatische Erfassen definierter Transponder kann der RFID-Handschuh in Verbindung mit der dahinterliegenden Softwarearchitektur den Nutzer durch seinen Arbeitsablauf navigieren. Wird bei einem Arbeitsschritt beispielsweise ein bestimmter, im Raum befindlicher RFID-Transponder gelesen, kann diese Tätigkeit im System als „abgeschlossen“ verbucht werden und die Ausgabe der Informationen für den folgenden Arbeitsschritt angestoßen werden. Die 
Identifikation des Transponders symbolisiert dabei die Bestätigung eines erfolgreich durchgeführten Arbeitsschrittes. Repräsentiert jeweils ein RFID-Transponder einen bestimmten Arbeitsschritt, kann der Nutzer mit Hilfe der während der Tätigkeiten unbewusst gelesen Transponder durch seinen gesamten Arbeitsablauf geführt werden.

\subsection{HMD zur Visualisierung der Informationen}

Für die Visualisierung prozessrelevanter Informationen existieren verschiedene, im industriellen Umfeld verbreitete Lösungen. Von einfachen Papierbelegen bis hin zu technisch ausgereiften Systemen wie Pick-byLight oder Pick-by-Voice sind gerade für die Kommissionierung verschiedene Systeme im täglichen Arbeitsumfeld erprobt. Im Bereich der manuellen Montage stellen beispielsweise stationäre (Touchscreen-) Terminals eine Alternative zur Bearbeitung der Aufträge anhand von papierbasierten Anleitungen dar. Vor allem in Bereichen, in denen der Mitarbeiter beide Hände zur freien Verfügung haben muss, können head-mounted Displays zur Optimierung der Arbeitsabläufe beitragen. Zur Prüfung und Kontrolle sicherheitsrelevanter Artikel im Wareneingang kann eine am Kopf getragene Datenbrille beispielsweise durchzuführende Arbeitsschritte oder Hinweise auf potenzielle Fehlerquellen direkt am Ort der Prüfung visualisieren. Ein weiteres Einsatzgebiet der Datenbrillen stellt die Wartung komplexer Anlagen dar. Durch eine aktive Interaktion über einen dezentralen Zugriff auf zentral abgelegte Daten können Mitarbeiter in Service oder Wartung vor Ort durch Mitarbeiter in der Zentrale unterstützt sowie Wartungs- oder Instandhaltungstätigkeiten beschleunigt und qualitativ verbessert werden. Die mitunter größten Potenziale bieten kopfbasierte Datenbrillen jedoch für die Bereiche Montage und Kommissionierung. Die Ursachen von Kommissionierfehlern sind beispielsweise häufig in einer schlechten Informationsbereitstellung begründet [Cro-07]. Über eine verbesserte visuelle Darstellung durch eine kontextbezogene Bereitstellung der Informationen zum aktuellen Prozessschritt und eine optische Hervorhebung des aktuellen Lagerplatzes können Fehler vermieden werden. Innerhalb von Montageprozessen können HMD helfen, eine hohe Variantenvielfalt zu beherrschen sowie bei der Bearbeitung komplexer Arbeitsanweisungen oder hochwertiger Produkte zu unterstützen. Wo kundenindividuelle Produkte hohe organisatorische Anforderungen an Produktion und Logistik stellen und prozessrelevante Informationen nur auf Umwegen bereitgestellt werden können, unterstützen Datenbrillen eine fehlerfreie Montage der Produkte durch die Visualisierung von Informationen am richtigen Ort (ausführliche Arbeitsanweisungen, Stücklisten, Explosionszeichnungen etc.).

\section{Gestaltung eines Systems zur papierlosen Produktion und Logistik}

Durch die Kombination eines mobilen RFID-Lesegerätes mit den Möglichkeiten der Augmented Reality wird es möglich, ein Systems zur flexiblen, papierlosen Bereitstellung, Visualisierung und Handhabung von Produktions- und Logistikdaten zu entwickeln. Grundlage für eine bedarfsgerechte Informationsbereitstellung über ein kopfbasiertes Display ist dabei die Berücksichtigung der technischen Rahmenbedingungen (RFID und $\mathrm{AR}$ ), die Integration ergonomischer Anforderungen (Mensch) sowie die empirische Erfassung und Strukturierung sämtlicher, für das System relevanter Daten (Prozess).

\subsection{Prozess}

Zur Qualifikation und Definition eines Einsatzszenarios für das System zur papierlosen Produktion und Logistik gilt es zunächst die Prozessabläufe in Abhängigkeit der technischen Rahmenbedingungen (Möglichkeiten und Grenzen) hinsichtlich der erreichbaren Einsatzpotenziale zu analysieren. Der für das System ausgewählte Prozess legt infolge dessen die zu erzielenden Leistungskennwerte (Prozesszeiten, Fehleranteile etc.) des Gesamtsystems fest. Zudem werden für die durchzuführenden Arbeitsabläufe sämtliche erforderliche Informationen, notwendige Interaktionen zwischen Mensch und Maschine sowie organisatorische Rahmenbedingungen determiniert. Letztere beziehen sich u. a. auf Systemanforderungen an die räumliche Umgebung oder die durch die technischen Einzelkomponenten zu gewährleistende Funktionsfähigkeit bedingt durch die erforderlichen Einsatzzeiten und Arbeitsbedingungen.

\subsection{Mensch}

Das für die papierlose Bereitstellung von Produktions- und Logistikdaten zentrale Element stellt der Mensch als Nutzer des Systems dar. Zur Steigerung der Nutzerakzeptanz ist es zwingend erforderlich, neben der physischen Belastung auch die psychische Belastung des Mitarbeiters, welche nach DIN ISO 10075 als „die Gesamtheit aller erfassbaren Einflüsse, die von außen auf den Menschen zukommen und psychisch auf ihn einwirken“ [DIN10075] definiert ist, sowie individuelle Parameter wie die Erfahrung des Mitarbeiters zu berücksichtigen.

(C) 2011 Logistics Journal: Not reviewed publications - ISSN 1860-5923

Seite 7 


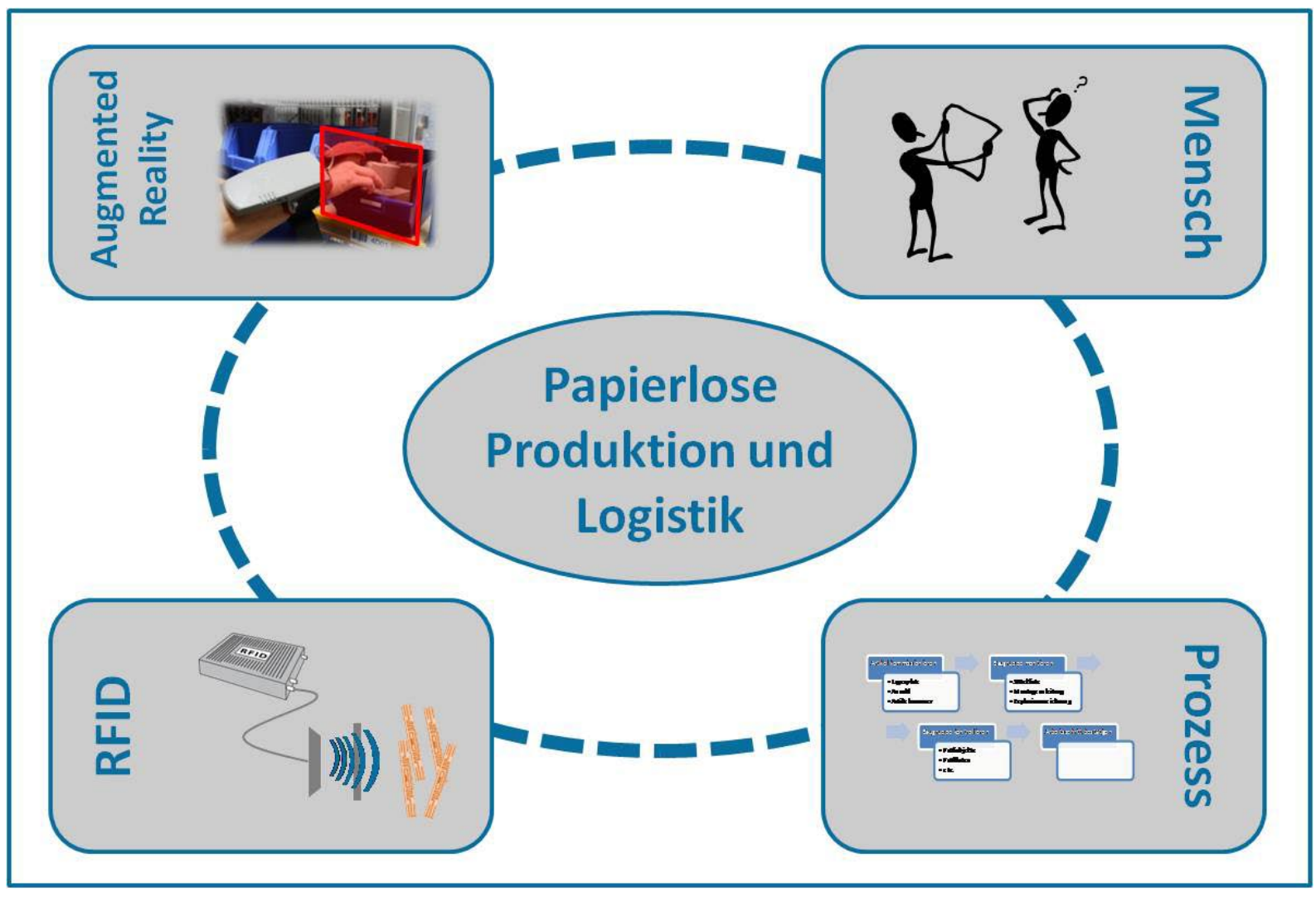

Abbildung 4: Einflussfaktoren auf das verfolgte Gesamtsystem

Eine Überforderung des Menschen durch eine suboptimale Bereitstellung der erforderlichen Informationen kann bspw. eine überhöhte psychische Belastung der Mitarbeiter verursachen. Eine erhöhte Fehlerhäufigkeit kann jedoch ebenfalls in der geistigen Unterforderung des Nutzers durch häufig wiederkehrende Tätigkeiten begründet sein. Eine gezielte Unterstützung des Mitarbeiters durch die Umsetzung der Grundsätze des Wearable Computing kann dabei helfen, Fehler in Arbeitsabläufen zu vermeiden.

\subsection{Augmented Reality}

Eine kontextabhängige Visualisierung prozessrelevanter Informationen wird im verfolgten System über den Einsatz eines Augmented Reality Systems realisiert, welches sich nach [Rei-09] in die Komponenten Datenhaltung, Visualisierungsmedium, Trackingsystem, Szenengenerator und Interaktionsgerät unterteilen lässt. Die Notwendigkeit der einzelnen Subsysteme, die jeweils spezifische Anforderungen an das Gesamtsystem stellen, hängt vom Umfang des eingesetzten AR-Systems ab. Wird das Ziel einer dynamischen Unterstützung der Blickführung des Nutzers verfolgt, stellen sich im Vergleich zur rein statischen Einblendung der Informationen deutlich höhere Anforderungen an das System. Für die Einblendung der virtuellen Objekte in der für den Nutzer richtigen Perspektive sind zusätzlich zum Visualisierungsmedium und Trackingsystem ein Szenengenerator sowie das damit verbundene Datenhaltungssystem erforderlich. Letzteres sucht die passenden Informationen zur jeweiligen Position des Nutzers und gibt diese an den Szenengenerator weiter [Alt-03]. Mobile bzw. stationäre Datenterminals stellen Alternativen für eine statische Visualisierung der Informationen dar.

\subsection{RFID}

Die aus dem RFID-System resultierenden Anforderungen definieren sich in erster Linie durch die Sicherstellung einer erfolgreichen Identifikation der Transponder, welche nahezu unbemerkt in die ursprüngliche Tätigkeit des Nutzers zu integrieren ist. Diese Tatsache stellt neben einer erforderlichen Mindestlesereichweite teils hohe Anforderungen an die Kompatibilität des RFID-Lesegerätes an individuelle Arbeitsabläufe (unterschiedliche Greifmuster, Arbeitsgeschwindigkeit etc.). Durch die Auswahl der im System verwendeten Transponder wird neben den für die Identifikation der Informationsträger erforderlichen Leistungsdaten auch die erreichbare Speicherkapazität festgelegt. Diese wiederum bestimmt die Struktur der Datenhaltung für das Gesamtsystem. Eine durchgängig dezentrale Datenhaltung auf dem Speicher des Transponders kann aufgrund beschränkter Kapazitäten meist nicht realisiert werden. Der Aufbau einer Datenbank sowie die Entwicklung eines kombinierten Datenhaltungssystems aus zentralen und dezentralen Komponenten sind dadurch unumgänglich. 
Gerade für Anwendungen in Wartung und Service können jedoch besonders die dezentral auf dem Transponder gehaltenen Daten von großem Interesse sein.

\section{Einsatzgebiete eines Systems zur papierlosen Produktion und Logistik}

\subsection{Unterstützung von Montageprozessen mit RFID und AR}

Die Perlenkettensteuerung gilt vor allem in der Automobilindustrie als erfolgsversprechende Umsetzung von Konzepten mit dem Ziel, die physische Reihenfolge der (Fahrzeug-) Aufträge aufrecht zu erhalten [Mei-09]. Bei ungeplanten Stillständen im Produktionsablauf oder unvorhersehbaren Schwankungen der geplanten Ausbringungsleistung vorgelagerter Fertigungsprozesse muss dabei jedoch sichergestellt werden, dass sich Produktion und Materialfluss weiterhin in einem Takt befinden. Zur Beherrschung von Veränderungen der SollAuftragsreihenfolge in Produktions- oder Montageprozessen kann ein RFID-gestütztes System Anwendung finden, welches den jeweiligen Auftrag sowie sämtliche prozessrelevanten Informationen dezentral auf einem Transponder am Produkt oder Behälter mitführt.

Das beschriebene System zur papierlosen Produktion und Logistik verfolgt den Ansatz, einen Behälter als Referenz zum zugeordneten Kommissionier- und Montageauftrag mit einem RFID-Transponder im UHFBereich zu kennzeichnen. Dieser Transponder beinhaltet Informationen zur Auftragsnummer sowie zum aktuellen Stand der Bearbeitung und stellt das Bindeglied für den Abruf zusätzlicher Informationen aus einer zentralen Datenbank dar. Werden in einer der Montage vorgelagerten Kommissionierung mehrere Aufträge anhand von mehreren Behältern parallel bearbeitet, kann bei der Abgabe eines Artikels in den Behälter zunächst überprüft werden, ob der Artikel korrekt abgelegt und somit dem richtigen Auftrag zugeordnet wurde. Bei einer fehlerhaften Abgabe kann über eine akustische Rückmeldung durch das mobile RFID-Lesegerät oder das am Kopf getragene Display ein Warnhinweis ausgegeben werden. Somit kann eine stabile Auftragsfolge durch eine korrekte Versorgung der nachfolgenden Montagetätigkeit sichergestellt und die Perlenkettensteuerung aufrecht erhalten werden. Zu Beginn der Montagetätigkeit kann der Transponder am auftragsspezifischen Behälter zudem genutzt werden, um die Visualisierung der relevanten Informationen zu den darauffolgenden Arbeitsschritten anzustoßen und den Nutzer somit durch den Prozess zu führen.

Bei Unsicherheiten im Bezug auf die korrekte Durchführung eines Montageschrittes ermöglicht die kontextbezogene Visualisierung über das head-mounted Display gerade für unerfahrene Mitarbeiter, auf zusätzliche Informationen aus einer zentralen Datenbank zurückzugreifen. Dazu ist neben einer kabellosen Schnittstelle zum zentralen Datenbank-Server ein zusätzliches Interaktionsgerät notwendig, um durch das bereitgestellte Hilfemenü zu navigieren. So können neben ausführlichen Beschreibungen des Arbeitsschrittes bspw. Stücklisten, Zeichnungen der einzelnen Bauteile oder Explosionszeichnungen bei der Bearbeitung des Produktes unterstützten. Für den Fall einer Sondervariante im Montageprozess, welche sich durch ein sehr geringes Auftreten von der gewöhnlichen Montage unterscheidet, kann ein Hervorheben von variantenspezifischen Informationen auf die Besonderheit hinweisen und somit eine fehlerhafte Bearbeitung verhindern.

\section{2. $\quad$ RFID-gestützter Prüfmechanismus in der Kommissionierung}

Neben der Unterstützung von Montageprozessen kann das mobile RFID-Lesegerät zudem zur Fehlervermeidung in der Kommissionierung eingesetzt werden. Auch ohne die Integration eines head-mounted Displays können die Grundfunktionalitäten des mobilen RFID-Lesegerätes genutzt werden, um die Entnahme von Artikeln abzusichern ohne den Werker dabei zu beeinträchtigen. Das gewohnte Informationsbereitstellungssystem (Pickby-Voice, Pick-by-Light, Kommissionierbeleg etc.) wird dabei lediglich um den RFID-Handschuh erweitert, der über eine kabellose Schnittstelle mit einer zentralen Recheneinheit verbunden ist und vom Mitarbeiter am Handgelenk getragen wird. Beim Griff in ein Lagerfach wird ohne zusätzlichen Aufwand für den Mitarbeiter ein dem Artikel zugeordneter Transponder erfasst. Dadurch ist es im Falle eines fehlerhaften Griffes möglich, direkt am Ort der Entnahme eine (akustische) Rückmeldung anzustoßen und somit auf den Fehler hinzuweisen. Die Zuordnung der Transponder zu den Artikeln kann dabei entweder über den jeweiligen Behälter, aber auch über den entsprechenden Lagerplatz erfolgen (vgl. Abbildung 5: Alternative Transponderanbringungen zur RFIDgestützten Kommissionierung). 


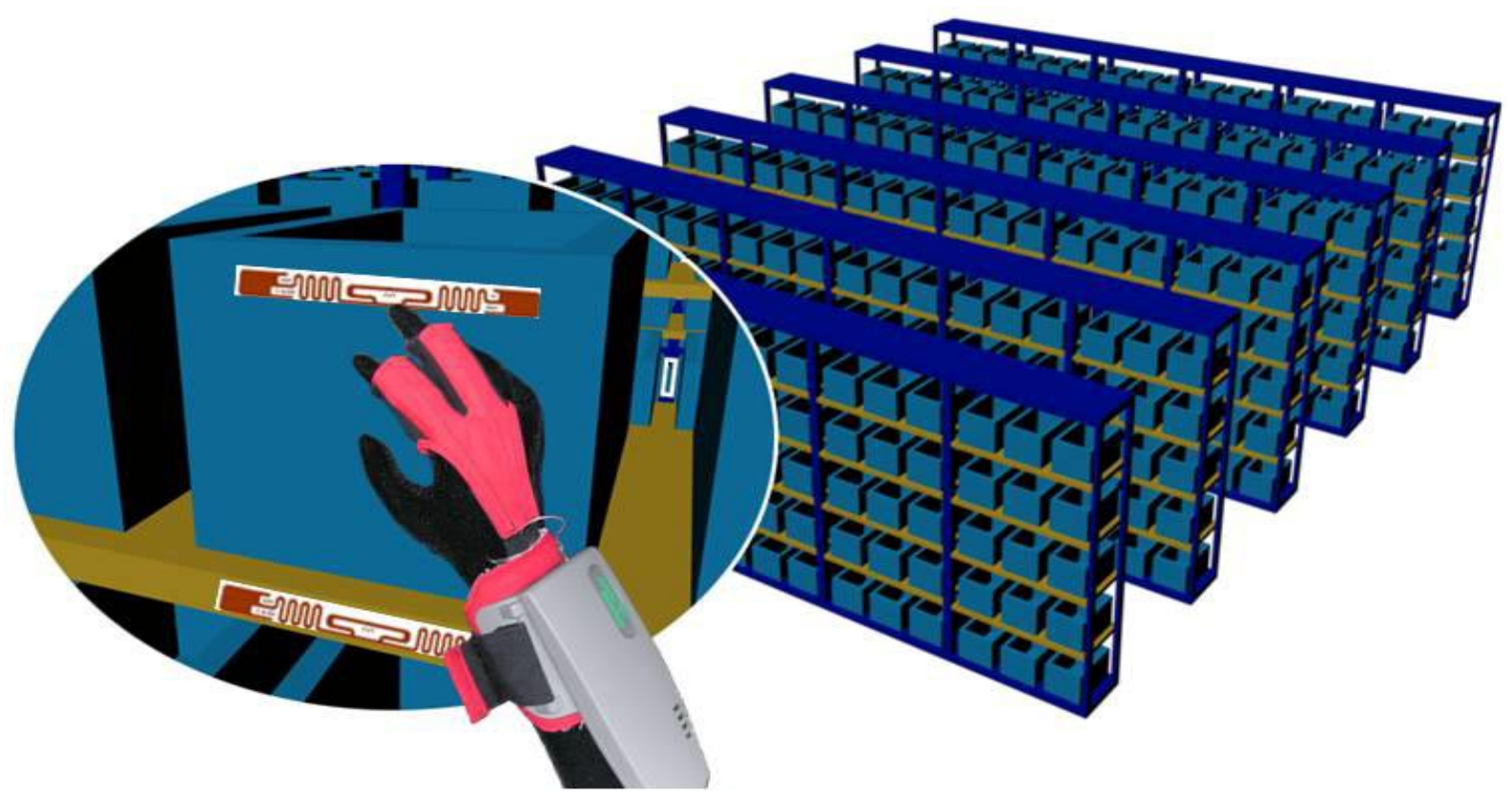

Abbildung 5: Alternative Transponderanbringungen zur RFID-gestützten Kommissionierung

Befinden sich die Behälter in einem offenen Kreislauf hat die Kennzeichnung der Lagerplätze mit RFIDTranspondern gegenüber der Behälterkennzeichnung Vorteile, da die Transponder lediglich in einem einmaligen Aufwand bei der Inbetriebnahme des System am Lagerplatz angebracht werden müssen. Die laufenden Kosten für die Kennzeichnung von Behältern durch stets neue Transponder sowie den damit verbundenen Aufwand zur immer wiederkehrenden Anbringung derselben würde die Rentabilität des Systems stark beeinträchtigen. Für beide Anbringungskonzepte gilt gleichermaßen, dass die Anpassung und Übertragbarkeit des Systems auf verschiedene Lagertypen (Fachbodenregal, Behälterlager, Lager für Großladungsträger etc.) gewährleistet ist.

Durch die Verwendung des mobilen RFID-Lesegerätes im Sinne des Wearable Computing als Prüfmechanismus kann der Nutzer dadurch auf etwaige Fehler aufmerksam gemacht werden. Erste Untersuchungen in industrieller Umgebung haben das Potenzial des Systems zur Vermeidung von Fehlern bei der Bereitstellung benötigter Teile bereits unter Beweis gestellt. Zudem wurde das System durch die Absicherung der Entnahmen parallel zur Kommissionierung anhand einer Papierliste von den Mitarbeitern als Arbeitserleichterung akzeptiert, obgleich zum Zeitpunkt der Untersuchung die Voraussetzungen zum Einsatz des RFID-Handschuhes in Produktivumgebungen nicht endgültig gegeben waren.

\section{7. $\quad$ Fazit}

Gerade in Produktion und Logistik wird der Mensch aufgrund seiner Flexibilität sowie sensomotorischen Fähigkeiten trotz einer zunehmend digitalisierten Welt auch in Zukunft ein wichtiger Baustein bleiben. Daher gilt es Konzepte und Lösungen für Mensch-Maschine-Schnittstellen aufzuzeigen, welche bei Bedarf den Eingriff des Menschen in eine virtuelle Welt aus vernetzten, intelligenten Objekten ermöglichen. Die Kombination aus einem mobilen, in den Arbeitsablauf integrierten RFID-Lesegerät und der Visualisierung relevanter Informationen über ein head-mounted Display kann dabei unterstützen. Innerhalb von Montageprozessen kann ein auftragsspezifischer RFID-Transponder an Behältern oder Produkten die kontextbezogene Bereitstellung der für den aktuellen Montageschritt relevanten Informationen anstoßen. Zudem kann der Transponder genutzt werden, um Daten dezentral am Produkt mitzuführen und somit auch für nachgelagerte Prozesse (Wartung, Service etc.) verfügbar zu machen. Aber auch den Einsatz von head-mounted Displays, welche aufgrund ergonomischer Defizite für eine industrielle Arbeitsumgebung aktuell nicht ausreichend entwickelt sind, kann ein RFID-gestütztes System nach den Grundsätzen des Wearable Computing Prozessabläufe optimieren. Der RFID-Handschuh kann dabei mit verschiedenen Kommissioniersystemen kombiniert werden. Beim Einsatz des Lesegerätes parallel zur Kommissionierung mit Etiketten und Papierlisten in industrieller Umgebung konnte das Potenzial zur Vermeidung von Fehlern bereits unter Beweis gestellt werden.

\section{Literatur}

[Aar-04]

[Alt-03]

(C) 2011 Logistics Journal: Not reviewed publications - ISSN 1860-5923
Aarts, E.: Ambient Intelligence: A Multimedia Perspective. In: Multimedia, IEEE, 2004.

Alt, T.: Augmented Reality in der Produktion. München: Herbert Utz Verlag, 2003.

Seite 10 
[Chr-03] Christ, O.; Fleisch, E.: Ubiquitous Computing: Von der Vernetzung von Computern zur Vernetzung von Dingen. In: Österle, H.; Winter, R.: Business Engineering. Auf dem Weg zum Unternehmen des Informationszeitalters. Berlin: Springer, 2003.

[Cro-07] Crostack, H.-A.; Deuse, J.: Optimierung von Kommissionierung und Verpackung durch geeignete Strategien für die Qualitätssicherung unter Berücksichtigung der Retourenabwicklung. Abschlussbericht zum Forschungsvorhaben, Dortmund, 2007.

[Did-10] Didier, M.: Arbeitswissenschaftliche Herausforderungen durch AmI. In: AmI und Arbeitswissenschaften - Chancen und Risiken neuer I\&K-Technologien in der Arbeitsumgebung, Tagungsunterlagen, 15.01.2010.

[DIN10075] DIN ISO 10075-1: Ergonomische Grundlagen bezüglich psychischer Arbeitsbelastung - Teil 1: Allgemeines und Begriffe. Berlin: Beuth Verlag, 2000.

[Fis-05] Fishkin, K.; Philipose, M.; Rea, A.: Hands-On RFID: Wireless Wearables for Detecting Use of Objects. In: Proceedings of the Ninth IEEE International Symposium on Wearable Computers (ISWC'05), 2005.

[Fle-01] Fleisch, E.: Business perspectives on Ubiquitous Computing. Institute of Information Management, University of St. Gallen, 30.11.2001.

[Fle-05] Fleisch, E.; Mattern, F.: Das Internet der Dinge - Ubiquitous Computing und RFID in der Praxis. Berlin, Heidelberg, New York: Springer-Verlag, 2005.

[Gün-09] Günthner, W. A.; Blomeyer, N.; Reif, R.; Schedlbauer, M.: Pick-by-Vision: Augmented Reality unterstützte Kommissionierung. Abschlussbericht zum Forschungsvorhaben, Garching, 2009.

[Ibe-09] Iben, H.; Baumann, H.; Klug, T.; Ruthenbeck, C.: Visual Based Picking Supported by Context Awareness - Comparing Picking Performance Using Paper-based Lists Versus List Presented on a Head Mounted Display with Contextual Support. In: ICMI-MLMI’09, Cambridge, MA, USA, 2009.

[IFF-10] http://www.iff.fraunhofer.de/iffdb_files/images/792_rfid_handschuh_foto_dirk_mahler_iff.jpg. (26.10.2010).

[Kir-10] Kirch, M.; Voigt, M.; Poenicke, O.; Richter, K.: Intelligente Infrastrukturen und Transportmittel zur kontinuierlichen Warenverfolgung. In: ISIS AutoID/RFID Special, 2010.

[Lam-05] Lampe, M.; Strassner, M.; Fleisch, E.: A Ubiquitous Computing Environment for Aircraft Maintenance. Institute of Technology Management, University of St. Gallen, 2005.

[Lee-10] Lee, C.; Kim, M.; Park, J.; Oh, J.; Eom, K.: Design and Implementation of the wireless RFID Glove for life applications. In: International Journal of Grid and Distributed Computing Vol. 3, No. 3, 2010.

[Lip-04] Lipp, L.: Interaktion zwischen Mensch und Computer im Ubiquitous Computing. Münster: Lit Verlag, 2004.

[Lit-10] http://www.liteye.com/product.html. (03.11.2010).

[Lus-07] Lustig, C.; Novatchkov, H.; Dunne, L.; McHugh, M.; Coyle, L.: Using Colocation to Support Human Memory. In: Workshop “Supporting Human Memory with Interactive Systems”, HCI Conference, Lancaster, UK, 2007.

[Mei-09] Meissner, S.: Logistische Stabilität in der automobilen Variantenfließfertigung. Technische Universität München, 2009.

[Phi-04] Philipose, M.; Fishkin, K.; Perkowitz, M.; Patterson, D.; Hähnel, D.: Inferring Activities from Interactions with Objects. In: PERVASIVE computing, IEEE, 2004.

[Rei-09] Reif, R.: Entwicklung und Evaluierung eines Augmented Reality unterstützten Kommissioniersystems. Dissertation, Technische Universität München, 2009.

[ScR-10] Scholz-Reiter, B.; Ruthenbeck, C.; Harjes, F.; Meinecke, Chr.: Informationsmanagement in der Logistik unterstützt durch Wearable Computing - Ein neuer Ansatz für das Informationsmanagement am Beispiel der Automobillogistik. In: Industrie Management, GITO-Verlag, 2010.

[ScA-00] Schmidt, A.; Gellersen, H.-W.; Merz, Chr.: Enabling Implicit Human Computer Interaction A Wearable RFID-Tag Reader. In: The Fourth International Symposium on Wearable Computers, IEEE, 2000.

[Sma-10] http://www.smart-TEC.com. (13.12.2010).

[Tüm-08] Tümler, J.; Mecke, R.; Doil, F.; Huckauf, A.; Urbina, M. H.; Roggentin, A.; Pfister, E. A.; Böckelmann, I.: Einbeziehung der Herzratenvariabilität für eine objektive Beanspruchungsanalyse beim Einsatz mobiler Augmented Reality an einem Referenzarbeitsplatz. In: 50. Fachausschusssitzung Anthropotechnik - Beiträge der Ergonomie zur Mensch-System-Integration. Deutsche Gesellschaft für Luft- und Raumfahrt - LilienthalOberth e.V., Manching, 22.-23.10.2008.

[VDI-10] http://www.vdi-nachrichten.com/vdi-nachrichten/aktuelle_ausgabe/ 
[Wei-91]

Weiser, M.: The Computer for the 21st Century. In: Scientific American, 1991.

[Wit-06]

Witt, H.; Kenn, H.: Towards Implicit Interaction by Using Wearable Interaction Device Sensors for more than one Task. In Proceedings of the 3rd International Conference on Mobile Technology, Applications and Systems (IEE Mobility Conference), Bangkok, Thailand, 2006.

[Woo-03] Wootley, S. I.; Cross, J. W.; Ro, S.; Foster, R.; Reynolds, G.; Baber, C.; Bristow, H.; Schwirtz, A.: Forms of wearable computer. Electronic, Electrical and Computer Engineering, The University of Birmingham, U.K., 2003. 